\title{
Investigation of the Biomechanic Function of Cruciate Ligaments Using Kinematics and Geometries from a Living Subject During Step Up/Down Motor Task
}

\author{
Luigi Bertozzi ${ }^{1}$, Rita Stagni ${ }^{2}$, Silvia Fantozzi ${ }^{2}$, and Angelo Cappello ${ }^{2}$ \\ ${ }^{1}$ Department of Electronics, Informatics and Computer Science, \\ University of Bologna Via Venezia 52, 47023 Cesena, Italy \\ lbertozzi@deis.unibo.it \\ ${ }^{2}$ Department of Electronics, Informatics and Computer Science, \\ University of Bologna Viale Risorgimento 2, 40136 Bologna, Italy \\ \{rstagni, sfantozzi, acappello\} @deis.unibo.it
}

\begin{abstract}
The modeling approach is the only possible way to estimate the biomechanic function of the different anatomical sub-structures of the knee joint in physiological conditions. Subject-specific geometry and kinematic data were the foundations of the 3D quasi-static model adopted for the present work. A previously validated cruciate ligaments model was implemented taking the anatomical twist of the fibers into account. The anatomical load components, developed by the modeled ligaments, were estimated during step up/down motor tasks. The anterior cruciate ligament never developed force, along every directions. The posterior cruciate ligament developed increasing forces with the increasing of the flexion angle until at about $70^{\circ}$ of flexion. Bigger repeatability in the force curves was obtained in extension with respect to the flexion movement. In conclusion the proposed model was effective in evaluating loads in the anterior and posterior cruciate ligament during the execution of daily living activities.
\end{abstract}

\section{Introduction}

In the human knee joint, the harmonious interaction among all its different anatomical sub-units provides the well known mobility and stability characteristics. The knowledge of the biomechanic function of the knee passive structures, like the cruciate ligaments, is of fundamental importance and of great clinical interest for the development of new effective rehabilitative and surgical procedures. This interest is demonstrated by over 8 million of injury related visits for knee symptoms by physicians and in emergency rooms, 381000 total knee replacements and 12000 other repair of cruciate ligaments performed in the USA in 2002 as reported by the American Association of Orthopedic Surgeons (AAOS) [1].

During its normal function, the knee lets the shank move with respect to the thigh, maintaining the stability under external articular load and torque. This is the result of several contributions: inter-segmental contact loads, ligaments tensioning, muscle forces, inertia of body segments. Thus, if we want to quantify the contribution of each anatomical structure, the only possible way is a modeling approach. 
The problem of the knee modeling has been tackled from different points of view and at different levels of complexity. Many two-dimensional models on sagittal plane were proposed in literature and several of these were based on a four-bar linkage modeling approach [2-6]. These models allowed to investigate the function of the knee ligaments only in the sagittal plane in different loading conditions $[7 ; 8]$. Threedimensional mathematical and finite elements models were also developed [9-12]. These can include sub-models of anatomical articular surfaces and contact forces, of articular deformations, of different passive structures, like ligaments, capsule and menisci, and of active structures like muscles. Nevertheless, these complex models were unusable in physiological context for their computational weight. The logical evolution of this approach could be the evaluation of a 3D model during the execution of a motor task characteristic of daily living activity [13;14]. In this context, even if the model is designed properly for a specific application, its potential can be nullified by the errors resulting from the anatomical, geometrical and mechanical parameters definition. In the cited papers $[2 ; 3 ; 7-9 ; 11-13]$, these errors were due to disagreement in the origins of parameters and inputs, which were often obtained from different and non-homogeneous sources.

Thus, in this work special attention was paid to the input data and parameter, in particular to the geometry influencing mechanics. Subject specific geometries and kinematic data are the foundations of the 3D quasi-static model adopted. The cruciate ligament models took the twisting of the fibers into account and the reference length of each fiber was estimated from the subject-specific passive flexion kinematics.

The aim of this study was the evaluation of the biomechanic role, in terms of forces, of the anterior and the posterior cruciate ligaments during a step up/down motor task.

\section{Material and Methods}

\subsection{Subject and Experimental Acquisitions}

The selected subject (male, height $168 \mathrm{~cm}$, weight $62 \mathrm{~kg}$, and age 30 years) underwent a high resolution nuclear magnetic resonance (NMR) scan of his right knee with a 1.5T Gemsow scanner (GE Medical Systems, Milwaukee, Wisconsin) [14], as reported in Table 1. The subject performed 2 repetitions of step up/down motor tasks while acquired by means of fluoroscopy (SBS 1600, Philips Medical System Nederland B.V.) at 10 images per second. The knee under analysis was kept inside the fluoroscopic field of view during the execution of the selected task. Moreover, for the

Table 1. The NMR scanning procedure parameters

\begin{tabular}{ll}
\hline Scanning sequence & Spin Echo $(\mathrm{T} 1$ weighted) \\
Number of slices & 54 \\
Pixel spacing & $0.037 \mathrm{x} 0.037(\mathrm{~cm} \cdot \mathrm{cm})$ \\
Scanned region length (across the knee) & $15.9(\mathrm{~cm})$ \\
Slice thickness & $2.5(\mathrm{~mm})$ \\
Slice spacing & $3(\mathrm{~mm})$ \\
\hline
\end{tabular}


detection of the subject specific fibers reference length [2;3], passive flexion was performed with the help of a qualified operator and acquired by means of the same fluoroscopic set-up.

\subsection{Knee Geometrical Model}

A 3D tiled surface geometrical representation of the distal femur, the proximal tibia, and the insertion areas of the anterior and posterior cruciate ligaments was generated from the NMR dataset using the software Amira (Indeed - Visual Concepts GmbH, Berlin, Germany). For each NMR slice, the outer contour of the structures of interest was detected and outlined with an entirely manual 2D segmentation technique. The resulting stacks of contours were interpolated to generate polygonal surfaces of each structure [14], as shown in Fig. 1(a-b).

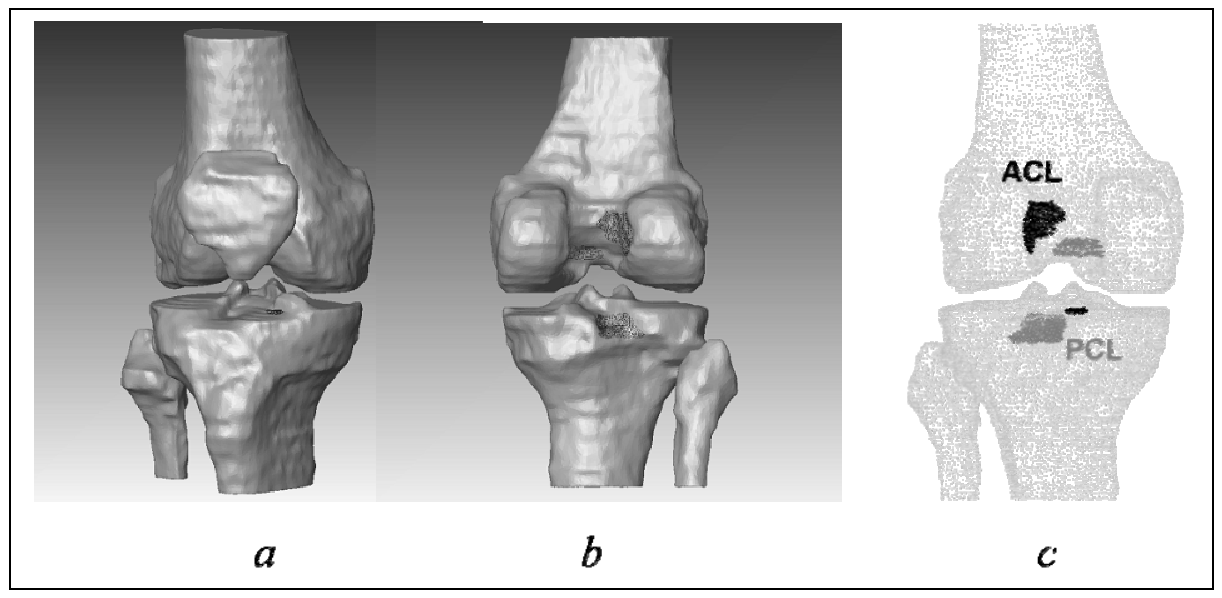

Fig. 1. Anterior and posterior view of the reconstructed bony geometries, $(a)$ and $(b)$ respectively. Anterior view of the bony geometries and the ligament insertion areas (dotted regions) on the femur and the tibia (c).

\subsection{Ligament Geometrical Model}

The anatomical insertion areas of both cruciate ligaments were described by a set of points. These were also calculated by means of the software Amira as prints of the ligament geometrical volume on the 3D bony surface, see Fig. 1(c). The inertia tensor was calculated from each cloud of points, and its principal axes and planes were calculated. The anatomical points were then projected on the first principal plane. A quadratic equation for each planar insertion area was estimated to fit the contour line of the projected anatomical points, and in each case an ellipse was obtained. The planar insertion points were then selected uniformly mapping 25 points on these elliptical areas. The 25 points were distributed: 1 in the centre of the ellipse, 12 uniformly distributed on the contour of the evaluated ellipse and 12 uniformly distributed along the contour of an ellipse having the same centre and semi-axes half of the previous one. The 25 planar insertion points selected on each elliptical area 
were then fitted on the 3D anatomical insertion area using the "thin plate splines" (TPS) method [15] as shown in Fig. 2(a).

The joining method between the femoral and tibial insertion points took the anatomical twist of the ligament fibers into account. The anterior cruciate ligament had the order of the fibers on the tibial insertion area rotated by $90^{\circ}$ laterally with respect to the femoral insertion area coherently with the anatomical external twist of the ligament, see Fig. 2(b). The posterior cruciate ligament had the order of the fibers on the tibial insertion area rotated by $90^{\circ}$ medially with respect to the femoral insertion area [16], see Fig. 2(c).

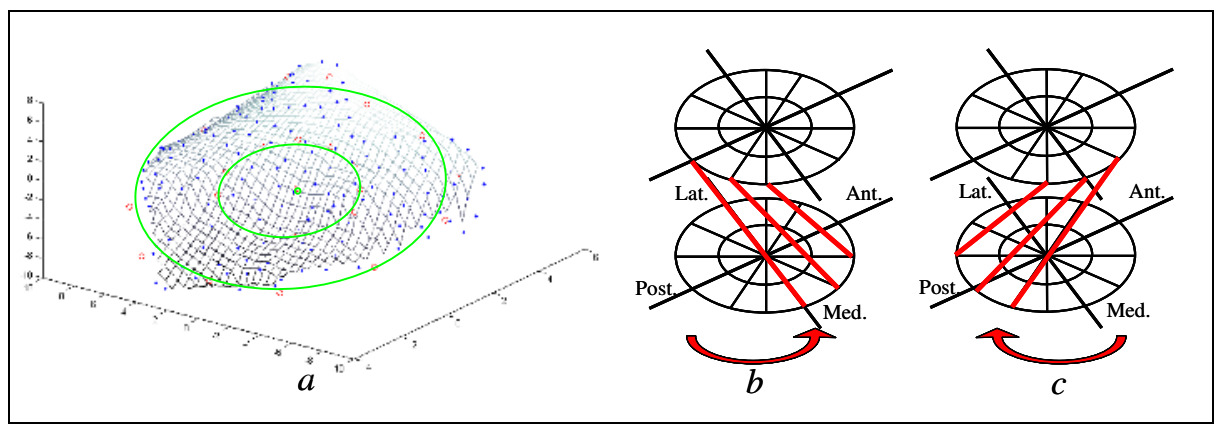

Fig. 2. A $3 D$ anatomical insertion area with the 25 fitted by TPS fiber insertion points mapped on two elliptical contours $(a)$; anterior $(b)$ and posterior cruciate ligament $(c)$ ordering pattern of the fibers

\subsection{Kinematics}

The accurate 3D bone pose in space was reconstructed by means of an iterative procedure using a technique based on tangency condition between the projection lines and the surface of the geometrical model. The accuracy of this technique was assessed to be 1 degree for rotations and $1 \mathrm{~mm}$ for translations [17]. The passive flexion and step up/down kinematics were then reconstructed.

\subsection{Mechanical Ligament Properties}

The two cruciate ligaments, in both models, were modeled with 25 different linearelastic elements. The elastic modulus $E$ of each ligament was the same and it was considered constant from literature equal to $175 \mathrm{MPa}$ [3]. The reference length $l_{0 j}$ of each fiber $j$ was defined according to Goodfellow's hypothesis [18], as the maximal length reached by each fiber during passive flexion. In a previous study, the authors validated this technique using the drawer test along the anterior and the posterior directions [19]. From the NMR dataset the total insertion area was known. The relative cross-sectional area $A_{j}$ for each fiber was calculated proportionally to the distance of each modeled insertion point from its adjacent ones after the TPS deformation on the anatomical surface.

The stiffness coefficient $K_{j}$ was calculated for each fiber $j$ with the equation (1) where $E, l_{0 j}$ and $A_{j}$ were the variables mentioned above. 


$$
K_{j}=\frac{E \cdot A_{j}}{l_{0 j}}
$$

The force expressed from each fiber was shown in equation (2) where $\Delta L_{j}$ was the difference between instant length $l_{j}$ and the reference length $l_{0 j}$ of the fiber.

$$
F_{j}=-K_{j} \cdot \Delta L_{j}
$$

The total ligament force was the vectorial sum of all fiber forces of the ligament. Obviously, the force expressed by each fiber was imposed to be zero if the distance between its two insertions was smaller than the reference length.

\subsection{Simulation and Post-processing Tools}

The mechanical system, composed from the bony geometries and the ligaments geometrical model including its mechanical properties, was implemented and animated with the acquired experimentally kinematics in ADAMS/View 2005 (MSC.Software Corporation 2 MacArthur Place Santa Ana,CA 92707 USA). This simulator of mechanical systems allowed to estimate each variable in the model, in particular, for each relative position between the femur and the tibia. The three components of the forces, anterior-posterior (A/P), proximal-distal (P/D) and mediallateral $(\mathrm{M} / \mathrm{L}) \mathrm{NMR}$ projections, and the magnitude for each fiber were calculated and exported for both cruciate ligaments. Post-processing elaborations were computed with Matlab 7 (The MathWorks, Inc, MA 01760-2098). The three components were set to zero when the magnitude of the force of each fiber was positive (compression), see equation (2). All these forces were transposed to the anatomical tibial reference system.

\section{Results}

The global qualitative behavior of the posterior cruciate ligament was very similar among the three anatomical directions, in particular considering the extension movements. In the $\mathrm{A} / \mathrm{P}$ and in the $\mathrm{M} / \mathrm{L}$ components similar and bigger forces were always reached than those reached in the P/D direction, see Fig. 3. The maximum forces reached were three times bigger in the extension movements and over five times bigger in the flexion movements.

In extension movements the mechanical contribution of the posterior cruciate ligament was very limited from the full extension to about $30^{\circ}-40^{\circ}$ of flexion. Then a rapid and quite linear increasing of its contribution was recorded until reaching the maximum force until about $70^{\circ}$ of flexion. At this angle of flexion a little decreasing of the forces was observed, in particular in the P/D direction, see in Fig. 3(b).

The behavior of the two flexion movements was different with respect to each other, in particular along the P/D direction. Like during the extension movements, also in this case, very little forces were expressed from the full extension to a variable angle at about $20^{\circ}-40^{\circ}$ of flexion. From this point, both two curves showed an increasing of their contribution until reaching bigger maximum forces with respect to 
those calculated in the extension movements. An isolated difference between the two curves was clearly evident in the P/D direction, where one of these began to decrease at about $45^{\circ}$ of flexion, see Fig. 3(e).

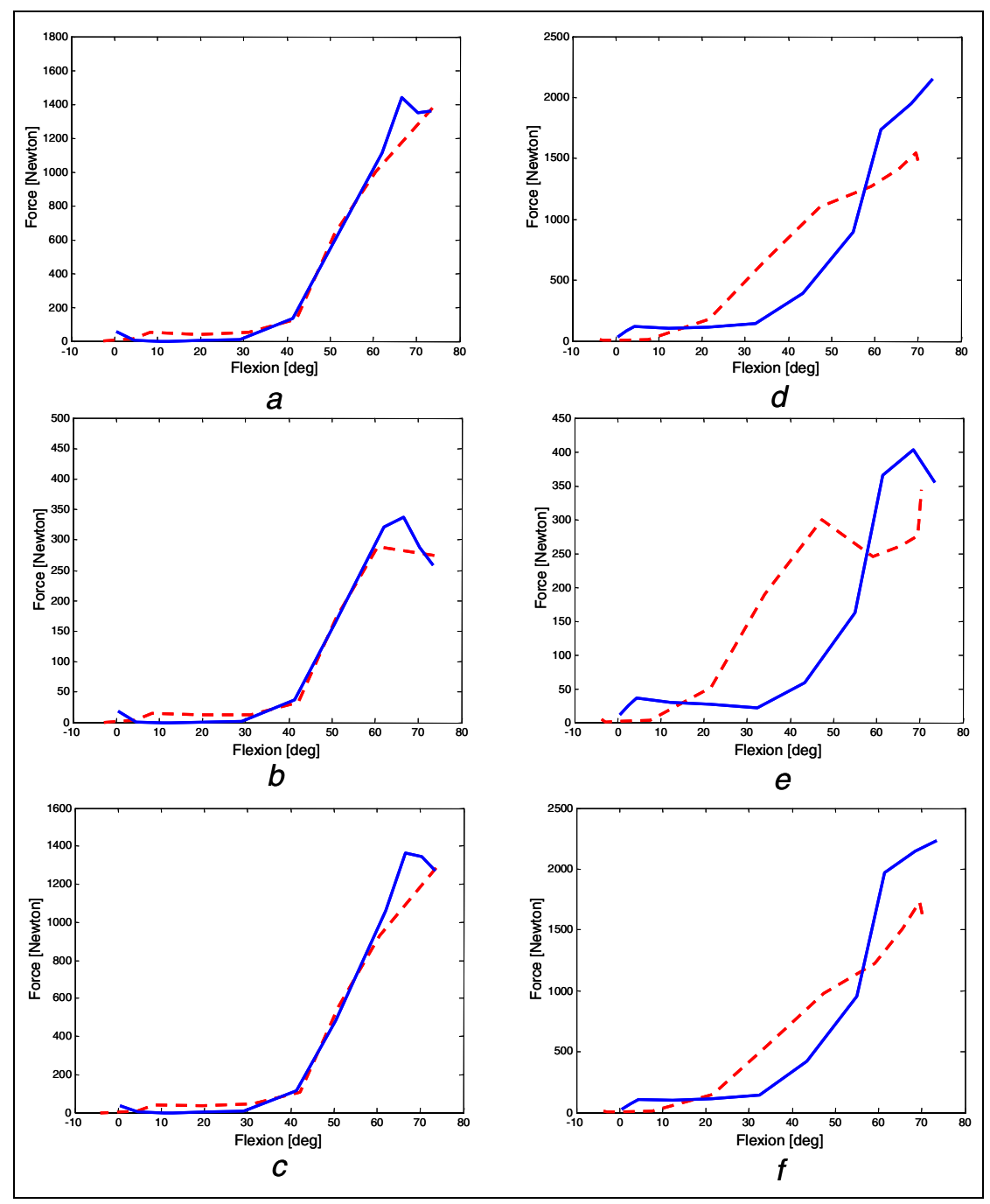

Fig. 3. Posterior cruciate ligament component forces on the anatomical tibial reference system versus the knee flexion angle ( 2 motor task repetitions: solid and dashed line): A/P, P/D and $\mathrm{M} / \mathrm{L}$ component forces of the three extension movements ( $a, b$ and $c$, respectively), and $\mathrm{A} / \mathrm{P}$, $\mathrm{P} / \mathrm{D}$ and $\mathrm{M} / \mathrm{L}$ component forces of the two flexion movements ( $d, e$ and $f$, respectively)

Regarding the anterior cruciate ligament the results obtained, during the simulations of both flexion and extension movements, were always equal to zero along every anatomical directions. 


\section{Discussion}

Anterior and posterior cruciate ligament models were implemented using geometrical parameters and kinematics data from a single living subject. Regarding the posterior cruciate, the greater repeatability obtained in the extension (step up movement) was probably due to a major activity of the muscles for controlling the movement. These had the goal to perform the movement against the gravity force and their concentric contractions were more controlled by the nervous system. On the other hand the flexion (step down movement) was an according to the gravity movement. Thus the eccentric contraction of the muscles was less controlled and a minor repeatability was obtained. The total inactivity of the anterior cruciate ligament was probably due to the typology of the movement that tends to slack the anterior cruciate and to stretch the posterior cruciate ligament. Indeed the biggest anatomical force components, regarding the posterior cruciate ligament, were obtained along the $\mathrm{A} / \mathrm{P}$ and the $\mathrm{M} / \mathrm{L}$ directions, where bigger contributions to the joint stabilization function were necessary.

Although the linearity of the mechanical characteristic assumed for each ligament fiber, the produced results were in agreement with physiology. In conclusion the proposed model, including all experimental acquisitions and data elaborations, was effective in evaluating subject-specific cruciate ligament loads during the execution of daily living activities.

\section{References}

1. AAOS. Internet site address: http://www.aaos.org

2. Zavatsky, A.B., O'Connor, J.J.: A model of human knee ligaments in the sagittal plane. Part 1: Response to passive flexion. Proc Inst.Mech.Eng [H.] 206 (1992) 125-134

3. Zavatsky, A.B., O'Connor, J.J.: A model of human knee ligaments in the sagittal plane. Part 2: Fibre recruitment under load. Proc Inst.Mech.Eng [H.] 206 (1992) 135-145

4. Gill, H.S., O'Connor, J.J.: Biarticulating two-dimensional computer model of the human patellofemoral joint. Clin Biomech 11 (1996) 81-89

5. Lu, T.W., O'Connor, J.J.: Lines of action and moment arms of the major force-bearing structures crossing the human knee joint: comparison between theory and experiment. $\mathrm{J}$ Anat 189 ( Pt 3) (1996) 575-585

6. Zavatsky, A.B., Wright H.J.: Injury initiation and progression in the anterior cruciate ligament. Clin. Biomech. 16 (2001) 47-53

7. Zavatsky A.B., O'Connor J.J.: Ligament forces at the knee during isometric quadriceps contractions. Proc. Inst. Mech. Eng [H.] 207 (1993) 7-18

8. Shelburne K.B., Pandy M.G.: A musculoskeletal model of the knee for evaluating ligament forces during isometric contractions. J. Biomech. 30 (1997) 163-176

9. Wismans J., Veldpaus F., Janssen J., Huson A., Struben P.: A three-dimensional mathematical model of the knee-joint. J. Biomech. 13 (1980) 677-685

10. Blankevoort L., Kuiper J.H., Huiskes R., Grootenboer H.J.: Articular contact in a threedimensional model of the knee. J. Biomech. 24 (1991) 1019-1031

11. Mommersteeg T.J., Huiskes R., Blankevoort L., Kooloos J.G., Kauer J.M.: An inverse dynamics modeling approach to determine the restraining function of human knee ligament bundles. J. Biomech. 30 (1997) 139-146 
12. Moglo K.E., Shirazi-Adl A.: Cruciate coupling and screw-home mechanism in passive knee joint during extension-flexion. J. Biomech. 38 (2005) 1075-1083

13. Piazza S.J., Delp S.L.: Three-dimensional dynamic simulation of total knee replacement motion during a step-up task. J. Biomech. Eng 123 (2001) 599-606

14. Stagni R., Fantozzi S., Davinelli M., Lannocca M.: Comparison of knee cruciate ligaments models using kinematics from a living subject during chair rising-sitting. Lecture Note in Computer Science Vol. 3036 Springer-Verlag (2004)1073-1080

15. Bookstein F.L.: Principal Warps: Thin-Plate Splines and the Decomposition of Deformations. IEEE Trans. Pattern Anal. Mach. Intell. 11 (1989) 567-585

16. Mommersteeg T.J., Kooloos J.G., Blankevoort L., Kauer J.M., Huiskes R., Roeling F.Q.: The fibre bundle anatomy of human cruciate ligaments. J. Anat. 187 Pt. 2 (1995) 461-471

17. Zuffi S., Leardini A., Catani F., Fantozzi S., Cappello A.: A model-based method for the reconstruction of total knee replacement kinematics. IEEE Trans. Med. Imaging 18 (1999) 981-991

18. Goodfellow J., O'Connor J.: The mechanics of the knee and prosthesis design. J. Bone Joint Surg. Br. 60-B (1978) 358-369

19. Bertozzi L., Stagni R., Fantozzi S., Cappello A.: 3D subject-specific model of the human knee from in-vivo measurements: validation on the knee drawer test. In submission to the Med. Eng. Phys. 